\title{
Finite Element Analysis of Aluminum Alloy Structural Hanger of Subway Vehicle
}

\author{
Xiaowei Yin ${ }^{1}$, Wenxue Qian ${ }^{2,}$, and Liyang Xie $^{2}$ \\ ${ }^{1}$ Department of Mechanical Engineering, Shenyang Institute of Engineering, \\ Shenyang 110136, China; \\ ${ }^{2}$ School of Mechanical Engineering and Automation, Northeastern University, \\ Shenyang 110819, China. \\ aqwx99@163.com
}

Keywords: Structural analysis, Finite element analysis, Aluminum alloy structure.

\begin{abstract}
Aluminum alloy structural hangers are widely used in subway vehicle. The advantage of aluminum alloy is light weight and corrosion resistance. For different application fields, the structures of aluminum alloy components are very different. They are typically lighter for the same strength and provide better heat conduction. As we know that do FEA (finite element analysis) is necessary before and after the alloy structures have been made. In this paper, a detail analysis was done with FEM (finite element method), and the stress distribution of alloy structural hanger was obtained. Also the FEA results show that the maximum stress is less than the yield stress and the stress concentration of the slot is relatively high.
\end{abstract}

\section{Introduction}

Aluminum alloy structural hanger usually is an important part of subway vehicle, and the failure of it can cause catastrophic results. Nowadays aluminum alloy structures are used more and more widely and usually they are made from an alloy of aluminum. They are typically lighter for the same strength and provide better heat conduction and improved cosmetic appearance. Once these aluminum casting improvements were more widely adopted, the aluminum alloy structure took its rightful place as low cost high performance. Energy, environment and security are the three issues of concern; it is also the three problems restricted the development of the car industry and the popularity of the car. The car safety and reliable depends largely on the performance of the aluminum alloy structure and service life. Aluminum alloy structure has many advantage such as light, heat dissipation faster, damping performance, long fatigue life, safe, reliable, beautiful exterior, precision size and easy to manufacture, and has a huge potential for development. The structure of aluminum alloy structure is complex, and when the automobile at work aluminum alloy structures bear centrifugal force and pressure of the automobile. Because there are stress concentrations, the true stress values of aluminum alloy structures are much greater than the nominal stress. It is useful for aluminum alloy structures to do stress and strain analysis detailed[1-4].

FEM (Finite element method) is a powerful method for structure analysis. The FEM originated from the need for solving complex structural analysis problems in civil and aeronautical engineering. Its history can be traced back to the work by Alexander Hrennikoff (1941) and Richard Courant (1942). While the approaches used by these pioneers are very different, they use one essential characteristic: mesh discretization of a continuous domain into a set of discrete sub-domains, usually called elements[5].

The finite element method began in earnest in the middle to late 1950s for airframe and structural analysis and gathered momentum at the University of Stuttgart through the work of John Argyris and at Berkeley through the work of Ray W. Clough in the 1960s for use in civil engineering By late 1950s, the key concepts of stiffness matrix and element assembly existed essentially in the form used today and NASA issued request for proposals for the development of the finite element software 
NASTRAN in 1965. The method was provided with a rigorous mathematical foundation in 1973 with the publication of Strang and Fix's An Analysis of The Finite Element Method, and has since been generalized into a branch of applied mathematics for numerical modeling of physical systems in a wide variety of engineering disciplines, such as thermodynamics and fluid dynamics.

Through FEA the stress levels and stress distributions of aluminum alloy structures can be obtained. After this has been done, it is easy to predict the static strength of aluminum alloy structures and furthermore it is a basic of dynamic analysis, fatigue prediction and reliability analysis to aluminum alloy structures[7-9].

\section{Finite element model}

The material of the structures is aluminum alloy. The grade of the aluminum alloy is AL6061-T6; the temperature of environment is $20^{\circ} \mathrm{C}$. The material parameters including elastic module, yield strength and Poisson ratio are shown in Table 1. The 3D model of the aluminum alloys structures and are shown in Figure 1. The structure of the hanger is very complex, and the best way is to build the structure as a parametric model, and then it is easy to analyze model response with different parameters of structures. In fact, the models are built in ProEngineering Wildfire 5.0, which is a world famous 3D modeling software. In ProEngineering Wildfire 5.0 it is very easy to change the sizes and shapes of the models. It is convenient to consider the response of hanger with different structures. Because there are geometric discontinuity at the bottom of slot and the rounds of the slots have stress concentration. In order to control the analysis error of FEA, the outline of hanger is meshed with brick element. Here a 20 -node element is used to obtain more accurate result. Fig. 1 is the 3D model of hanger. Figure 2 is the meshed model of hanger. Because the hanger has a structure of symmetry, only half of it needs to be built in FEM model.

Table 1 Linear elastic analysis material parameters of hanger

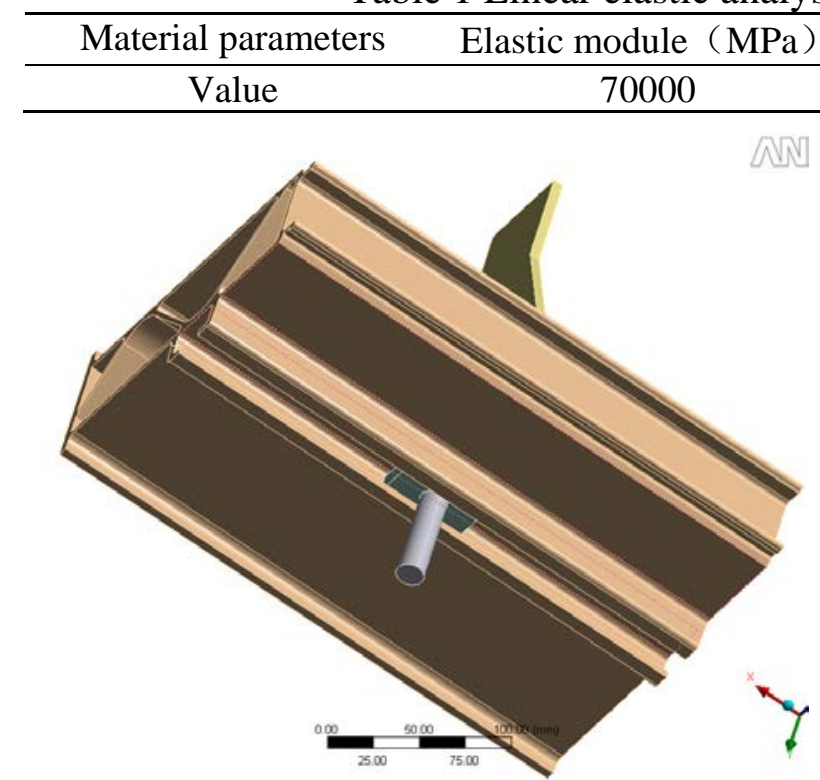

Fig.1 3D model of alloy hanger

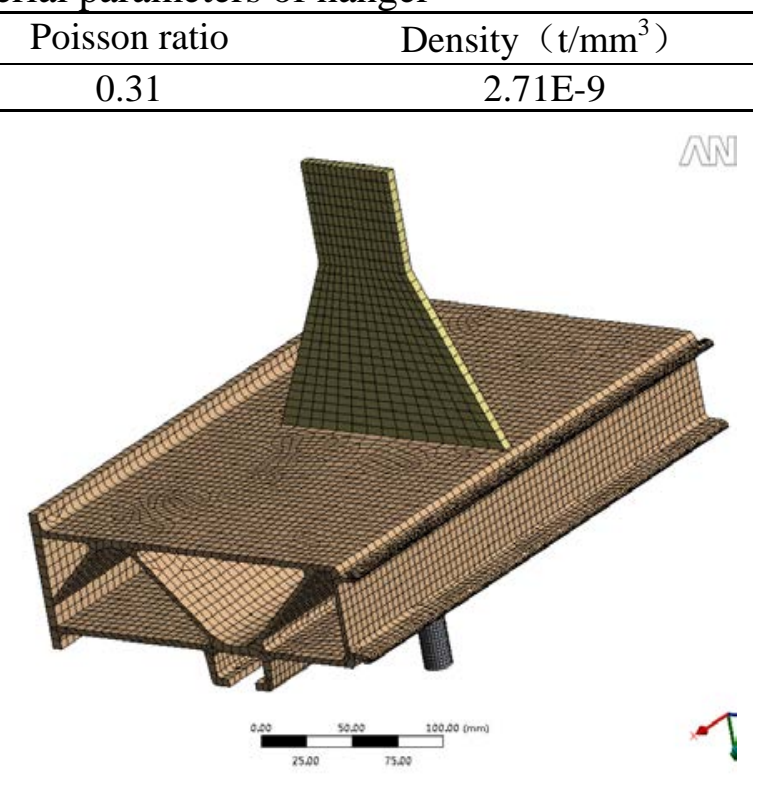

Fig.2 Meshed model of alloyhanger

\section{Structural strength analysis}

The major load of hanger is the tensile at the loading end, so after define the element type, material properties and load; we can do analysis with FEM. After we do a FEA of aluminum alloys structures, we get the stress level and distribution. The stress distributions of hangers are shown in Figure 3. From Fig.4 we can see that there are two locations the stress levels are relative high. One location is near the bottom of the slot and the other location is at the round of the ribs. 
Figure 4 is the deformation distribution of the hanger. The maximum stress is $228.36 \mathrm{MPa}$, which is less than the yield strength limit. That means the round of the mortise is not yield. To hanger at work, the slot of it will in elastic state, of cause after a long period there will emerge fatigue cracks. Fatigue crack fracture is a main type that the hanger fails. From the FEA results, the node No. of maximum stress is 148756 . The yield stress of the hanger is $240 \mathrm{MPa}$, so the fatigue of hanger is high cycle fatigue.

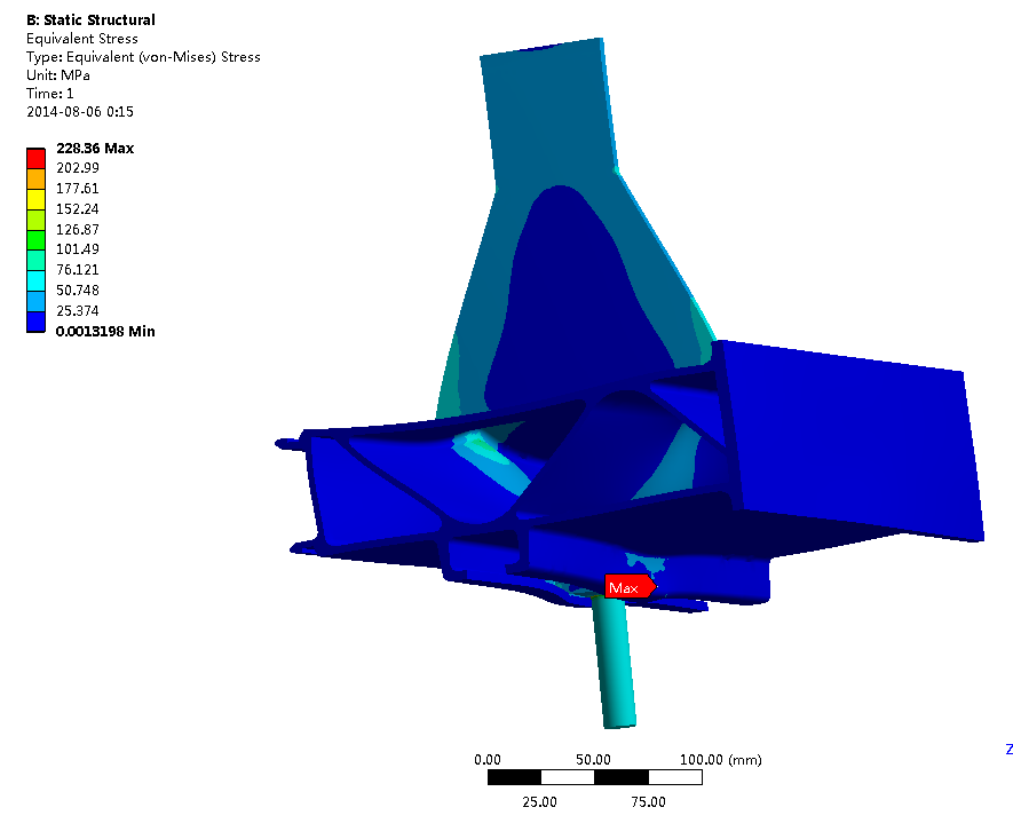

Fig.3 Stress distribution of alloy hanger

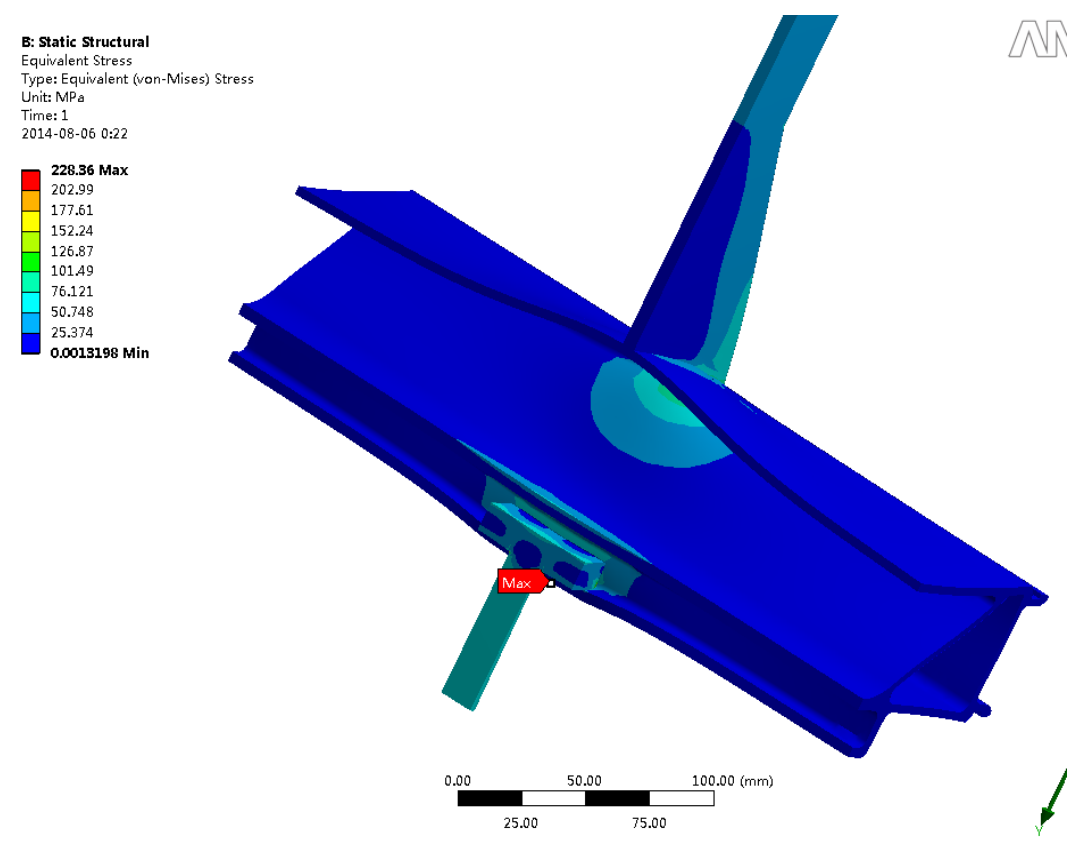

Fig.4 Section view of alloy hanger

\section{Conclusion}

Nowadays alloy hanger is used more and more widely for its excellent behaviors. Here an alloy aluminum alloy structural hanger is analyzed and the stress distribution is obtained. Through above analysis, it can be seen that the stress of slot round is the largest stress and it is the most dangerous location. The results show that the largest stress of the hanger is less then yield stress and the working safety factor is much less than the allowed safety factor. That means the hanger is safety enough and 
has a high reliability. Also it can be seen that the safety analysis and structure analysis are necessary and useful.

\section{Acknowledgments}

This work was partially supported by the National Natural Science Foundation of China (Grant No. 51005044, 51305275, 51335003, 51275221), and the Fundamental Research Funds for the Central Universities (Grant No. N140301001).

\section{References}

[1] Gottfried Dana A, Fleeter Sanford, Turbo machine blade row interaction predictions with a three-dimensional finite element method, Journal of Propulsion and Power, 18(2002) 978-989.

[2] Fujita M, Rackwitz R, Updating First and Second Order Reliability Estimates by Importance Sampling, Struct. Eng. Earthquake Eng. 5 (1988) 31 - 37.

[3] Wenxue Qian, Xiaowei Yin, Liyang Xie, Dayan Huang, Intelligent Analysis Method of Mechanical Structure Performance, International Forum on Information Technology and Applications, 2 (2009) 113 - 116.

[4] Qian Wenxue, Yin Xiaowei, Xie Liyang, Complex System Reliability using Bayesian Networks, Proceedings of the 15th International Conference on Industrial Engineering and Engineering Management, A ( 2008) 176-179.

[5] Shi J, Andrew P.D., Finite element modelling and analysis of a ceramic matrix composite combustor, Ceramic Engineering and Science Proceedings, 1998, 19 (4): 225-232.

[6] Qian Wenxue, Yin Xiaowei, Xie Liyang, System Reliability Allocation Based on Bayesian Network, Applied Mathematics \& Information Sciences, 6 (2012) 681-687.

[7] Ciarlet, Phillippe G., The Finite Element Method for Elliptic Problems. Amsterdam: North-Holland, 1978.

[8] Waterman, Pamela J., Meshing: the Critical Bridge, Desktop Engineering Magazine, 2008.

[9] Qian Wenxue, Yin Xiaowei, Xie Liyang, Reliability Modeling and Assessment of Component with Multiple Weak Sites under Complex Loading, Mathematical Problems in Engineering, 2014, Article ID 583803, 9 pages. 